\title{
Further COVID-19 Infection Control and Management Recommendations for the ICU
}

\author{
Robert A. Raschke MD \\ HonorHealth Osborne Medical Center \\ Scottsdale, AZ USA
}

An ad hoc committee of intensivists from the Phoenix area has been meeting via Zoom. They are sharing some of their thoughts and recommendations. Like the previous ICU recommendations published in SWJPCC (1), these are not necessarily evidence-based but based on recent experience and published experience with previous coronavirus outbreaks such as SARS. They are meant to supplement CDC recommendations, not to conflict or restate them.

\section{Infection control outside the rooms of suspected/confirmed COVID-19 patients.}

1. All healthcare workers should be allowed to exercise droplet precautions at all times while at work.

2. All staff should wear a single surgical mask per day to see all non-COVID patients and for rounds. The mask mitigates droplet spread bidirectionally between patients and HCWs and also helps prevent inadvertent touching of the nose and mouth.

3. Treat all code patients with airborne / standard / contact precautions

4. Use MDIs in preference to SVNs (as long as MDls hold out)

5. Reduce unnecessary staff and visitor traffic in all patient rooms. Avoid duplication of effort, repeated chest examinations with a stethoscope, in the same day by various doctors and nurses, are unlikely to benefit the care of most patients. Don't enter the patient's room without a specific purpose and try to perform multiple required tasks with each room entry.

6. Hand washing before/after: doorknobs, eating, using a computer, phones, googles.

7. Phones - use your own cellphone rather than shared landlines. Use speaker phone so you don't have to touch your face.

8. Consolidate computer use temporally and geographically. Clean your entire workstation (keyboard, mouse, surrounding desktop) before and after each use.

9. Keep track of and clean any object on your person that might be contaminated with fomites. This includes any medical instruments that you touch will your gloved hands while seeing patients (stethoscope, pen light, googles, etc). Leave these at work.

10. When walking down hallways, don't touch things.

\section{Patients under investigation or with known COVID-19.}

1. Avoid use of high-flow nasal cannula (HFNC) or BiPAP. This in opposition to surviving sepsis campaign recommendations, but data from SARS-CoV-1 show that non-invasive ventilation was associated with increased risk of infection of health care workers (2).

2. Use metered dose inhalers (MDIs) instead of small volume nebulizers (SVNs). 
3. Use N95 or PAPR during aerosol-producing procedures such as obtaining nasopharyngeal swab for SARS-CoV-2 RT-PCR, HFNC, BiPAP, bronchoscopy, intubation, breaking ventilator circuit for any reason, extubation, tracheostomy.

4. Consider early intubation. Prepare the bag mask with a high-efficiency particulate air (HEPA) filter and attempt rapid sequence intubation with fiberoptic laryngoscope.

5. If available, powered air-purifying respirators (PAPR) using P100 HEPA filters (filter $>99.97 \%$ of 0.3 um particles) should be considered over N-95 (filter $95 \%$ of 5 um particles) masks during this high risk procedure based on prior reports of SARS CoV-1 transmission to health care workers (HCW) wearing N95 masks PAPR protects the entire head and neck of the HCW, but requires additional training on donning/duffing.

6. If unable to wear PAPR, we recommend N95 masks, gowns and gloves, with googles instead of open face shielded masks. Aerosolized particles are more likely to pass around shields into eyes during these high-risk procedures. Also recommend hats and foot protection.

7. The smallest number of personnel required to safely perform the intubation should be present in the room. Fiberoptic laryngoscopy may be preferred over direct laryngoscopy to reduce exposure to aerosolized particles.

8. Once intubated:

a. Be sure all connections in the ventilator circuit are tight and do not break the circuit casually.

b. Place HEPA filter on exhalational limb of ventilator.

c. Obtain bronchial secretions using closed-circuit suction device

Code blue patients.

1. Use the same precautions as for COVID-19 patients in all patients for whom a code is called.

2. We recommend aerosol, contact and standard precautions and eye protection for all code team members for all codes - regardless of whether COVID-19 is suspected. There is no time in a code to determine the likelihood the patient has COVID-19, and bag-masking and intubation will aerosolize the patient's respiratory secretions.

3. A HEPA filter should be placed between the patient and the bag mask to reduce aerosolization of viral particles into the atmosphere.

\section{Diagnosis of COVID-19.}

The sensitivity of RT-PCR for COVID-19 is currently uncertain, but preliminary data suggests it may only be in the range of $70 \%$ for nasopharyngeal swabs and respiratory secretions. Bronchoscopy with bronchoalveolar lavage may have sensitivity about $90 \%$, but likely poses a risk to HCWs. This poses difficulty in ruling-out COVID-19. Bayesian logic dictates that the pre-test probability of disease influences interpretation of test results.

During active epidemic in Wuhan, the prevalence of COVID-19 among patients admitted with suspicion of having viral pneumonia was $60 \%$ (3). Assuming sensitivities by RTPCR for NP swab of $70 \%$, respiratory secretions $70 \%$, and BAL $90 \%$, and specificity 
$>95 \%$, the false negative rate for a single NP swab used to rule out COVID-19 is $31.6 \%$ - that is, $31.6 \%$ of patients taken out of isolation based on the negative NP swab result would actually be infected with COVID-19. If a second test, for instance respiratory secretions or another NP swab were performed on all patients whose first test was negative, the false negative rate for the series of tests is $8.8 \%$ - likely still not good enough to rule a patient out with confidence. If the second test was a BAL, the false negative rate for the series is $3.5 \%$.

In patients with high pre-test probability of COVID-19, a negative NP swab PCR cannot be safely relied-upon to rule out COVID-19. We recommend bronchial secretions be sent for PCR (in addition to NP swab) in all suspected patients who are intubated. A negative CT scan reduces the probability that a hospitalized patient has COVID-19, but will uncommonly be "negative" in hospitalized patients in whom the diagnosis is considered.

\section{Infection control at home during a surge.}

1. Clothes: don't wear jewelry/watches. Wear hospital-laundered scrubs at work, or take off your work clothes when you get home and throw them in wash machine. Leave your work shoes in your car.

2. Work equipment: Leave stethoscope, pen, googles and other work-related equipment in a locker at work. Wash your hands and ID badge just before getting in your car to leave the hospital. Leave your ID badge in your car while away from work. Don't bring your personal computer into work unless absolutely necessary.

3. Food: Put a Purell dispenser in front of the refrigerator. Stay out of the kitchen. If have your food prepared for you. Eat on paper plates and then throw them out yourself.

4. Use separate bathroom and sleeping quarters if available.

\section{References}

1. Raschke RA, Till SL, Luedy HW. COVID-19 prevention and control recommendations for the ICU. Southwest J Pulm Crit Care. 2020;20(3):95-7. [CrossRef]

2. Cheng VC, Chan JF, To KK, Yuen KY. Clinical management and infection control of SARS: lessons learned. Antiviral Res. 2013 Nov;100(2):407-19. [CrossRef] [PubMed]

3. Wang W, Xu Y, Gao R, Lu R, Han K, Wu G, Tan W. Detection of SARS-CoV-2 in different types of clinical specimens. JAMA. 2020 Mar 11. [Epub ahead of print] [CrossRef] [PubMed] 